\title{
Yeast extract inhibits the proliferation of renal cell carcinoma cells via regulation of iron metabolism
}

\author{
DAEUN MOON ${ }^{1,2}$, JINU KIM ${ }^{1}$ and SANG-PIL YOON ${ }^{1,3}$ \\ ${ }^{1}$ Department of Anatomy, School of Medicine; ${ }^{2}$ Interdisciplinary Graduate Program in Advanced Convergence \\ Technology and Science; ${ }^{3}$ Institute for Medical Science, Jeju National University, Jeju 63243, Republic of Korea
}

Received December 17, 2018; Accepted July 15, 2019

DOI: $10.3892 / \mathrm{mmr} .2019 .10593$

\begin{abstract}
The microbiome has recently attracted research interest in a variety of subjects, including cancer. In the present study, it was determined that reinforced clostridium media (RCM) for microbiome culture, exerts antitumor effects on renal cell carcinoma cells when compared to the microbiome ' $\mathrm{X}$ '. The antitumor effects of RCM were investigated for all ingredients of RCM, and the results revealed that yeast extract could be a candidate for the ingredient driving this phenomenon. Further experiments including MTT assay, cell counting, cell death analysis, cell cycle analysis and western blotting were conducted with yeast extract on renal cell carcinoma cells (Caki-1 and Caki-2) and normal human proximal tubular cells (HK-2). As a result, yeast extract exhibited dose-dependent antitumor effects on Caki-1 and Caki-2, but only slight effects on HK-2. In addition, yeast extract only exhibited slight effects on necrosis, autophagy, or apoptosis of Caki-1 and Caki-2. Yeast extract produced cell cycle arrest with an increased $\mathrm{G}_{0} / \mathrm{G}_{1}$ fraction and a decreased $\mathrm{S}$ fraction, and this was considered to be related to the decreased cyclin D1. Although yeast extract treatment increased anti-oxidant activities, the antitumor effects of yeast extract were also related to iron metabolism, based on the decreased transferrin receptor and increased ferritin. In addition, decreased GPX4 may be related to iron-dependent cell death, particularly in Caki-2. These results revealed that yeast extract may inhibit proliferation of renal cell carcinoma cells by regulating iron metabolism. Since an increased iron requirement is a classic phenomenon of cancer cells, yeast extract may be a candidate for adjuvant treatment of renal cell carcinoma.
\end{abstract}

Correspondence to: Professor Sang-Pil Yoon, Department of Anatomy, School of Medicine, Jeju National University, 102 Jejudaehak-ro, Jeju 63243, Republic of Korea

E-mail: spyoon@jejunu.ac.kr

Key words: yeast extract, renal cell carcinoma, iron, cell cycle, ferroptosis

\section{Introduction}

Renal cell carcinoma ( $\mathrm{RCC}$ ), the most common type of kidney cancer, originates from the proximal tubule (1), and an increasing incidence of 21.7 per 100,000 people has been observed over last decade as well as a 5-year survival rate of $79.9 \%$ in South Korea (2). The most common initial treatment of RCC involves removing the affected kidney, and then multiple therapies are used, including medications when metastasis occurs. However, RCC is known to be resistant to chemotherapy and radiotherapy in most cases (3).

There has been substantial interest regarding the use of microbiota in cancer research. Although in vitro and animal model studies suggest a protective anticancer effect of probiotics, the results of human epidemiological studies are still controversial $(4,5)$. In chronic kidney disease or end-stage renal disease patients, a correlation has been observed between the chronic alteration of intestinal microbiota homeostasis, dysbiosis, and chronic kidney disease $(6,7)$. Although the relationship among gut microbiota-derived metabolites, signaling pathways, and kidney diseases remains to be elucidated, gut microbiota-derived short-chain fatty acids have been revealed to be involved in kidney diseases through the activation of the gut-kidney axis. The main beneficial effects of short-chain fatty acids on kidney function involved decreasing inflammation and enhancing antioxidant activity (8). Furthermore, dysbiosis could promote many diseases, including colonic and extracolonic cancers (4). Microorganism fermentation extract has exhibited a growth inhibitory effect on cancer cells (9).

With the increasing interest in the use of gut microbiota in the extra-intestinal field, the effects of microbiota on preventive or therapeutic modality in kidney cancer were investigated. In our preliminary study, an unknown microbiome-X and the reinforced clostridial media (RCM) for microbiota culture as a positive control were used to treat RCC and human kidney proximal tubular cells (HK-2). RCM unexpectedly exhibited anticancer effects compared with the microbiome-X, thus the potential growth inhibitory activity of RCM was studied on primary (Caki-2) and metastatic (Caki-1) RCC cell lines.

\section{Materials and methods}

Cell culture. The HK-2 human kidney proximal tubular cell lines [American Type Culture Collection (ATCC)] 
and Caki-1 and Caki-2 clear cell RCC cell lines (Korean Cell Line Bank) were respectively cultured in RPMI-1640 medium and McCoy's 5A, both supplemented with $10 \%$ fetal bovine serum (FBS; Welgene, Inc.) at $37^{\circ} \mathrm{C}$ with $5 \% \mathrm{CO}_{2}$, as previously described (10) as indicated by ATCC. For the anchorage-dependent culture, each cell was seeded in a cell culture dish (90x20 mm; SPL Life Sciences).

Reagents and antibodies. Difco ${ }^{\mathrm{TM}}$ reinforced clostridial medium (RCM) was purchased from BD Biosciences and all ingredients assessed including yeast extract were obtained from Sigma-Aldrich; Merck KGaA (Table I). 3-[4,5-Dimethylthiazol-2-yl]-2,5-diphenyl tetrazolium bromide (MTT) was purchased from Amresco, Inc. (VWR Inernational LLC).

The antibodies used were specific for caspase-3 (diluted 1:2,000; product no. 9662) and cleaved caspase-3 (diluted 1:500; product no. 9661; both from Cell Signaling Technology, Inc.), c-Myc (diluted 1:1,000; cat. no. sc-764) and catalase (diluted 1:5,000; cat. no. sc-271803; both from Santa Cruz Biotecnology, Inc.), cyclin B1 (diluted 1:1,000; product no. 4138) and cyclin D1 (diluted 1:2,000; product no. 2978; both from Cell Signaling Technology, Inc.), ferritin heavy chain (FTH1; diluted 1:2,000; cat. no. sc-376594) and GAPDH (diluted 1:5,000; cat. no. sc-25778; both from Santa Cruz Biotecnology, Inc.), glutathione peroxidase 4 (GPX4; diluted 1:1,000; ID product code ab41787; Abcam), LC3-I/II (diluted 1:2,000; product no. 12741; Cell Signaling Technology, Inc.), p21 (diluted 1:1,000; cat. no. 60214-1-Ig; Proteintech Group, Inc.), SLC7A11 (cysteine/glutamate transporter (xCT); diluted 1:2,000; cat.no. ANT-111; Alomone Labs), SOD-1 (Cu-ZnSOD; diluted 1:5,000; cat. no. sc-11407), SOD-2(MnSOD; diluted 1:5,000; cat. no. sc-30080) and transferrin receptor (CD71, TfRC; diluted 1:2,000; cat. no. sc-65882; all from Santa Cruz Biotecnology, Inc.).

Cell counting and MTT assay for cell viability. Cells (5x10 $/$ each cell line) were seeded in appropriate media supplemented with $10 \%$ FBS, washed twice with phosphate-buffered saline (Welgene, Inc.), and then fresh medium was added. Next, various concentrations of RCM $(0.5,1.0,5.0$, and $10.0 \%)$ and yeast extract $(1,5$, and $10 \%$ dissolved in distilled water (DW) prior to the experiment) were added to the cells. The number of viable cells was estimated at various time-points (up to $72 \mathrm{~h}$ of culture) using trypan blue staining, as previously described (11), since an MTT assay revealed interference when treated with high concentrations of RCM and yeast extract.

The effect of the ingredients of RCM on cell viability was evaluated using MTT reduction into its formazan product as instructed by the manufacturer. Cells $\left(2 \times 10^{3} /\right.$ each cell line $)$ were seeded in triplicate wells in 96-well plates and treated with each ingredient and RCM itself. Next, the cells were incubated for $72 \mathrm{~h}$ and then MTT reagent $(5 \mathrm{mg} / \mathrm{ml}$ in PBS) was added into each well for $2 \mathrm{~h}$, dissolved in DMSO for $15 \mathrm{~min}$, and the MTT reduction was assessed spectrophotometrically at 595 and $620 \mathrm{~nm}$ as background using a VERSAmax microplate reader (Molecular Devices Korea LLC). The absorbance values obtained from the wells of the vehicle (DW)-treated cells represent $100 \%$ cell viability and were used for comparisons with the treated cells.
Flow cytometry. Cells were treated with or without $5.0 \%$ yeast extract for $72 \mathrm{~h}$. For cell death analysis, suspended cells were incubated with $5 \mu \mathrm{l}$ Annexin V-FITC and $5 \mu \mathrm{l}$ propidium iodide for $15 \mathrm{~min}$ at room temperature in the dark using the EzWay Annexin V-FITC Apoptosis Detection Kit (KOMA Biotech) according to the manufacturer's protocol. Biding buffer was added to each mixture, and the samples were analyzed through flow cytometry within $1 \mathrm{~h}$ using the FACSCalibur ${ }^{\mathrm{TM}}$ system (BD Biosciences).

For cell cycle analysis, the cells were fixed in $70 \%$ ethanol for $1 \mathrm{~h}$ at $4^{\circ} \mathrm{C}$, washed with PBS, and treated with $100 \mu \mathrm{g} / \mathrm{ml}$ RNase A (Sigma-Aldrich; Merck $\mathrm{KGaA}$ ) for $1 \mathrm{~h}$ at $37^{\circ} \mathrm{C}$. Next, the cells were stained with $25 \mu \mathrm{g} / \mathrm{ml}$ propidium iodide (Sigma-Aldrich; Merck KGaA) for $15 \mathrm{~min}$ at $37^{\circ} \mathrm{C}$. Flow cytometry was then performed using the FACSCalibur ${ }^{\mathrm{TM}}$ system (BD Biosciences) and analyzed by BD FACStation software version 6.0 (BD Biosciences), as previously described (11).

Western blotting. In order to obtain intracellular proteins, cultured cells were harvested in M-PER mammalian protein extraction reagent (Thermo Fisher Scientific, Inc.) including $1 \%$ protease inhibitor cocktail set III (EMD Millipore), $0.5 \%$ phosphatase inhibitor cocktail 2 and $0.5 \%$ phosphatase inhibitor cocktail 3 (both from Sigma-Aldrich; Merck KGaA). Protein concentration was assessed using BCA protein assay (Thermo Fisher Scientific, Inc.) according to the manufacturer's instructions.

The electrophoresis of protein in cell lysates on any TGX Stain-Free FastCast ${ }^{\mathrm{TM}}$ Acrylamide Starter Kit (Bio-Rad Laboratories, Inc.) using tris/glycine buffer systems (product nos. 161-0772 and 161-0771; Bio-Rad Laboratories, Inc.) onto PVDF membranes was performed as previously described (10).

The membranes were first blocked with 5\% skim milk for $1 \mathrm{~h}$ and then incubated with primary antibodies overnight at $4^{\circ} \mathrm{C}$. After washing, peroxidase anti-mouse or anti-rabbit IgG antibodies (cat. no. WB-2000 or WB-1000; Vector Laboratories, Inc.) were applied for $1 \mathrm{~h}$ at room temperature. Next, western lighting chemiluminescence reagent (product no. NEL101; PerkinElmer, Inc.) was used to detect proteins. The anti-GAPDH antibody was used as a loading control on the stripped membranes. The bands were quantified using AzureSpot analysis software (version 14.2; Azure $^{\mathrm{TM}}$ c300; Azure Biosystems, Inc.).

Statistical analysis. All data were compiled from a minimum of three replicate experiments. Data are expressed as the mean \pm standard deviation. The results were compared between treated and control cells using Student's t-test (SPSS version 14.0) and compared among groups or cell lines using ANOVA with a Bonferroni post-hoc test (both from SPSS, Inc.). P $<0.05$ was considered to indicate a statistically significant difference.

\section{Results}

Yeast extract is a candidate for the reinforced clostridium media (RCM)-related viability on RCC cells. It was revealed that when compared to the microbiome ' $\mathrm{X}$ ', $\mathrm{RCM}$ had antitumor effects on RCC cells. In our preliminary study, the MTT assay was not a useful method due to interference, and the results were compared with the cell counting results (data not shown). 
Table I. List of the ingredients of Difco ${ }^{\mathrm{TM}} \mathrm{RCM}$ formula and used reagents in the experiments.

Used ingredient

(cat. no., purchased

from Sigma-Aldrich;

Difco $^{\mathrm{TM}}$ RCM formula (/liter)

Merck KGaA)

\begin{tabular}{lrc}
\hline Agar & $0.5 \mathrm{~g}$ & $\mathrm{~A} 1296$ \\
Beef extract & $10.0 \mathrm{~g}$ & - \\
Cysteine $\mathrm{HCl}$ & $0.5 \mathrm{~g}$ & $\mathrm{C} 1276$ \\
Dextrose & $5.0 \mathrm{~g}$ & $\mathrm{D} 9434$ \\
Peptone & $10.0 \mathrm{~g}$ & - \\
Sodium acetate & $3.0 \mathrm{~g}$ & $\mathrm{~S} 2889$ \\
Sodium chloride & $5.0 \mathrm{~g}$ & - \\
Soluble starch & $1.0 \mathrm{~g}$ & $\mathrm{~S} 9766$ \\
Yeast extract & $3.0 \mathrm{~g}$ & $\mathrm{Y} 1625$ \\
\hline
\end{tabular}

RCM, reinforced clostridial medium.

RCM inhibited the growth of all cells examined in a dose-dependent manner under cell counting. Following treatment with various concentrations of $\operatorname{RCM}(0.5,1.0,5.0$, and $10.0 \%$ ) for $72 \mathrm{~h}$, the HK-2 cell viability was reduced to 96.65 , $83.73(\mathrm{P}=0.018), 63.83(\mathrm{P}=0.001)$, and $35.58 \%$ of that of the vehicle-treated condition, respectively. The viability of Caki-1 and Caki-2 were respectively reduced to $67.43(\mathrm{P}<0.01)$, 46.92, 17.06, and 2.76\% and $77.63(\mathrm{P}=0.031), 45.48(\mathrm{P}=0.008)$, 19.03 , and $5.94 \%$ of those of the vehicle-treated condition, respectively (Fig. 1A).

In a subsequent experiment, a dose of $1.0 \%$ RCM was used. All ingredients except for peptone and beef extract were assessed for the antitumor effects of RCM using MTT assay. Compared to the counting results, HK-2, Caki-1, and Caki-2 cells exhibited relatively high ratios of viable cells at $1 \% \mathrm{RCM}$ treatment, with values of 83.73 vs. $118.67,46.92$ vs. 68.12 , and 45.48 vs. $62.17 \%$, respectively (Fig. 1B). Sodium acetate $(\mathrm{P}=0.048)$, starch $(\mathrm{P}=0.028)$, and yeast extract $(82.40 \%$ vs. the vehicle, $\mathrm{P}=0.001)$ revealed considerable antitumor effects on Caki-2, but all of the ingredients had only slight effects on Caki-1, with the lowest viability with yeast extract (91.92\% vs. the vehicle, $\mathrm{P}=0.202$ ).

Yeast extract exhibits dose-dependent and time-dependent antitumor effects on RCC cells. Following $72 \mathrm{~h}$ of incubation, it was observed that yeast extract exerted antitumor effects on the RCC cells in a dose-dependent manner at 1.0, 5.0 and $10.0 \%$ concentrations by volume compared to the normal kidney proximal tubular cells (HK2; $\mathrm{P}=0.194)$ (Fig. 1C).

Yeast extract did not affect HK-2 cells, in which the viability was counted as $92.04 \%$ even with $10 \%$ yeast extract treatment $(\mathrm{P}=0.018)$. Yeast extract exhibited significant antitumor effects in a dose-dependent manner): viabilities of 79.11-41.01\% and 78.16-49.12\% at $1.0-5.0 \%$ yeast extract treatments on Caki-1 and Caki- 2 cells, respectively. Accordingly, the expected $\mathrm{IC}_{50}$ was $5.0 \%$ of yeast extract on RCC cells. Therefore, a dose of $5.0 \%$ yeast extract was used in all subsequent experimentation.
With $5.0 \%$ yeast extract, time-dependent antitumor effects were estimated in RCC cells (Fig. 1D). Compared to Caki-1, Caki-2 exhibited relatively slow proliferation. Caki-1 and Caki-2 exhibited decreased cell viability in a time-dependent manner: after $72 \mathrm{~h}$, the ratios of proliferation were $42.88 \%$ (2.81 times vs. 1.19 from the $24-\mathrm{h}$ incubation) and $51.54 \%$ (2.11 times vs. 0.96 from the 24 -h incubation) of the control levels in Caki-1 and Caki-2, respectively.

Yeast extract does not affect the cell death of RCC cells. In order to investigate the mechanism responsible for the antitumor effects of yeast extract, cell death and related protein levels were evaluated.

Yeast extract induced necrosis and apoptosis and were identified through Annexin V/PI staining. Compared to the control, yeast extract resulted in no marked increase in the rate of necrosis or apoptosis in RCC cells (Figs. 2A and 3A). Although statistically significant differences were observed for late apoptosis $(0.09-0.36 \%, \mathrm{P}=0.005)$ and necrosis $(0.73-1.70 \%, \mathrm{P}=0.011)$, the percentage was extremely small in the case of Caki-2 (Fig. 3A).

Furthermore, the expression of apoptosis-related caspase-3 (total vs. cleaved form) and autophagy-related LC3 I/II was detected (Figs. 2B and 3B). Total caspase-3 was significantly decreased $(\mathrm{P}=0.047)$ in Caki-1, but in the other proteins was unchanged following yeast extract treatment. Accordingly, the expression of caspase-3 was not significantly altered in Caki-1 $(\mathrm{P}=0.219)$ or Caki-2 $(\mathrm{P}=0.283)$. Both LC3 I and LC3 II were significantly decreased in Caki- $1(\mathrm{P}<0.001$ and $\mathrm{P}=0.001$, respectively) and increased in Caki-2 $(\mathrm{P}=0.005$ and $\mathrm{P}=0.008)$, however the expression level of LC3 II/I was sustained under yeast extract treatment.

Yeast extract affects the cell cycle of RCC cells. Yeast extract inhibited the proliferation of RCC cells. The effects of yeast extract on the cell cycle were assessed through PI staining. Both Caki-1 (Fig. 4A) and Caki-2 (Fig. 5A) cells were incubated with $5 \%$ yeast extract, revealing a significant increase in the $\mathrm{G}_{0} / \mathrm{G}_{1}$ phase $(\mathrm{P}=0.019$ and $\mathrm{P}=0.036$, respectively) and a decrease in the $\mathrm{S}$ phase $(\mathrm{P}=0.015$ and $\mathrm{P}=0.033$, respectively).

Compared to the untreated control cells, the fraction of Caki-1 and Caki-2 cells in the $\mathrm{G}_{0} / \mathrm{G}_{1}$ phase demonstrated a significant upward trend (4.64\% Caki-1, 20.38\% increase Caki-2) following treatment with $5 \%$ yeast extract. Specifically, the $\mathrm{G}_{0} / \mathrm{G}_{1}$ fractions were 78.92 and $75.65 \%$ in untreated cells and 83.56 and $96.03 \%$ in cells treated with yeast extract in Caki-1 and Caki-2, respectively.

In order to delineate the mechanisms underlying the cell cycle arrest induced by yeast extract, p21, cyclin D1, cyclin B1, and c-Myc were assessed, which all promote cell cycle progression. Under yeast extract treatment, the expression of cyclin D1 $(\mathrm{P}=0.008)$ and $\mathrm{c}-\mathrm{Myc}(\mathrm{P}=0.015)$ was significantly decreased in Caki-1 (Fig. 4B), while those of p21 ( $\mathrm{P}=0.007)$, cyclin D1 ( $\mathrm{P}=0.017)$, and cyclin $\mathrm{B} 1(\mathrm{P}<0.001)$ were significantly decreased in Caki-2 (Fig. 5B).

Yeast extract differentially affects intracellular antioxidant activity in RCC cells. Following yeast extract treatment, potential changes in the intracellular antioxidant activity were investigated in RCC cells (Fig. 6). The levels of catalase, 

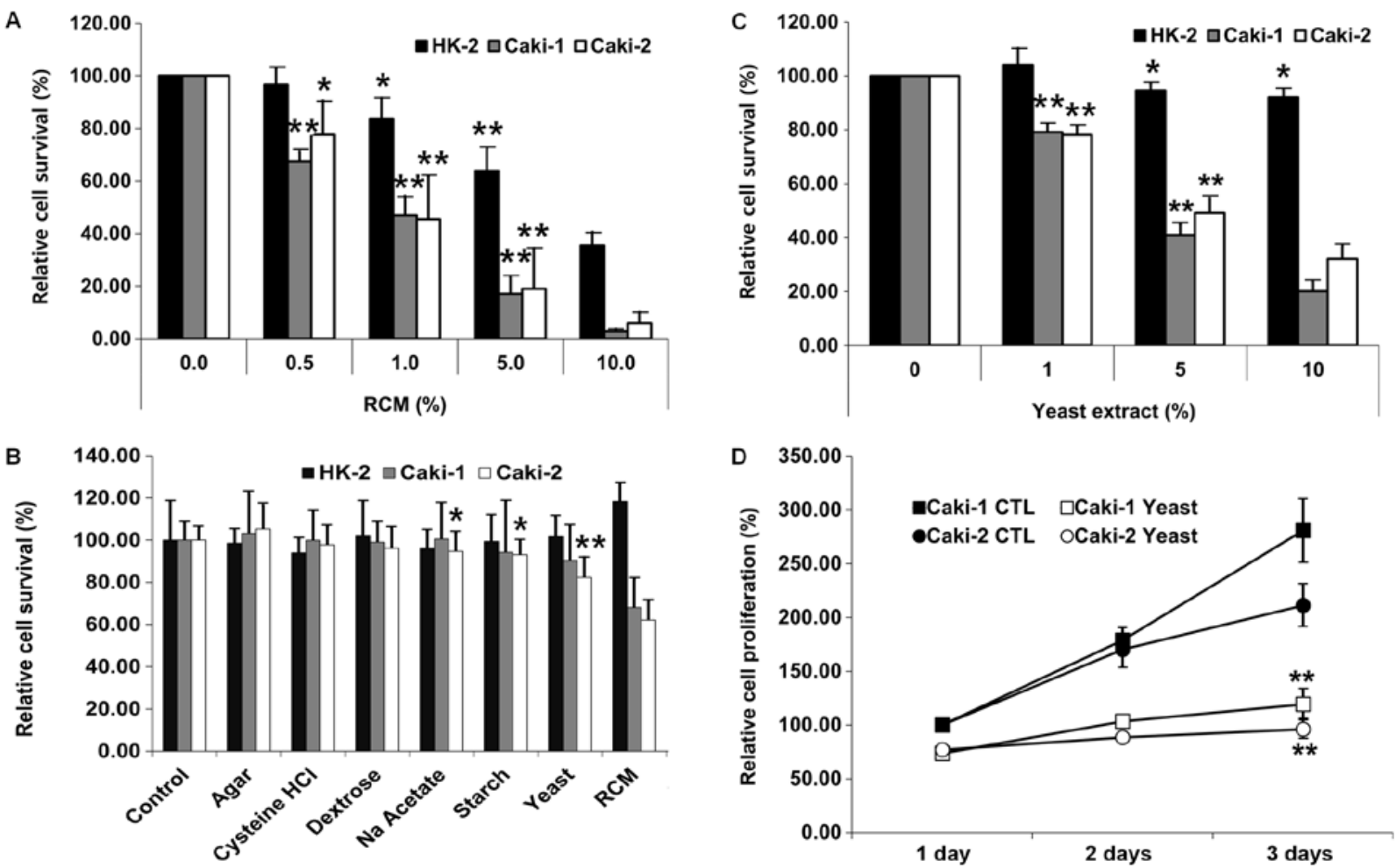

Figure 1. Yeast extract is a candidate for the antitumor effects of RCM on RCC cells. (A) RCM exhibited dose-dependent antitumor effects on RCC cells (Caki-1 and Caki-2) following $72 \mathrm{~h}$ of incubation, but slight effects on the normal kidney proximal tubular cells (HK-2). (B) Yeast extract was a candidate for the antitumor effects of all subunits of RCM. An MTT assay was not a useful method since it exhibited interference (RCM), as compared with the cell counting results of A. (C) Yeast extract exhibited dose-dependent antitumor effects on the RCC cells compared to the normal kidney cells following $72 \mathrm{~h}$ of incubation. Anticipated $\mathrm{IC}_{50}$ was $5.0 \%$ of yeast extract on RCC cells, and this was used in all subsequent experimentation. (D) Yeast extract (5.0\%) significantly inhibited the proliferation of metastatic (Caki-1) and primary (Caki-2) RCC cells. Cell survival is presented vs. the survival of vehicle-treated cells following treatment with yeast extract. Data are expressed as the mean \pm standard deviation. ${ }^{*} \mathrm{P}<0.05$ and ${ }^{* *} \mathrm{P}<0.01$ vs. the vehicle-treated control (A-C) or data from $1 d$ (D). $\mathrm{RCM}$, reinforced clostridial media; RCC, renal cell carcinoma.
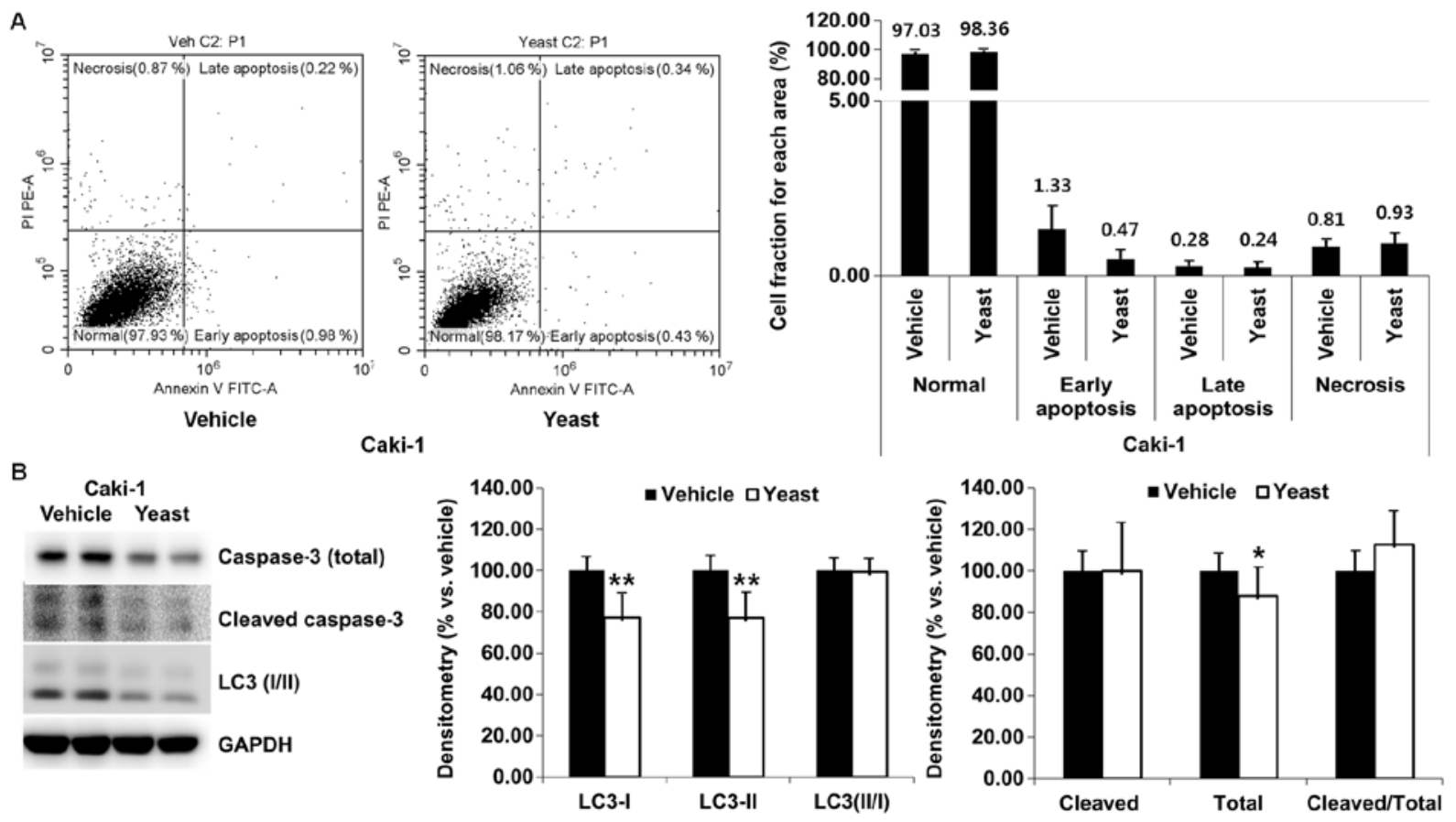

Figure 2. Yeast extract does not affect cell death signaling of metastatic RCC cells (Caki-1). (A) Annexin V/PI staining was used to assess the necrosis and apoptosis induced by $5.0 \%$ yeast extract in Caki-1 cells. Representative results of flow cytometric analysis of cell death distribution, in which the percentage of each quadrant is numerically indicated. Quantitative data are expressed as the mean \pm standard deviation, which were not significant for necrosis and apoptosis. (B) Western blot analysis revealed caspase-3-dependent apoptosis and LC3 dependent autophagy. There were no significant increases in cell death pathways. Densitometry is presented vs. the vehicle-treated cells following treatment with yeast extract. Data are expressed as the mean \pm standard deviation. ${ }^{*} \mathrm{P}<0.05$ and ${ }^{* *} \mathrm{P}<0.01$ vs. vehicle. $\mathrm{RCC}$, renal cell carcinoma. 

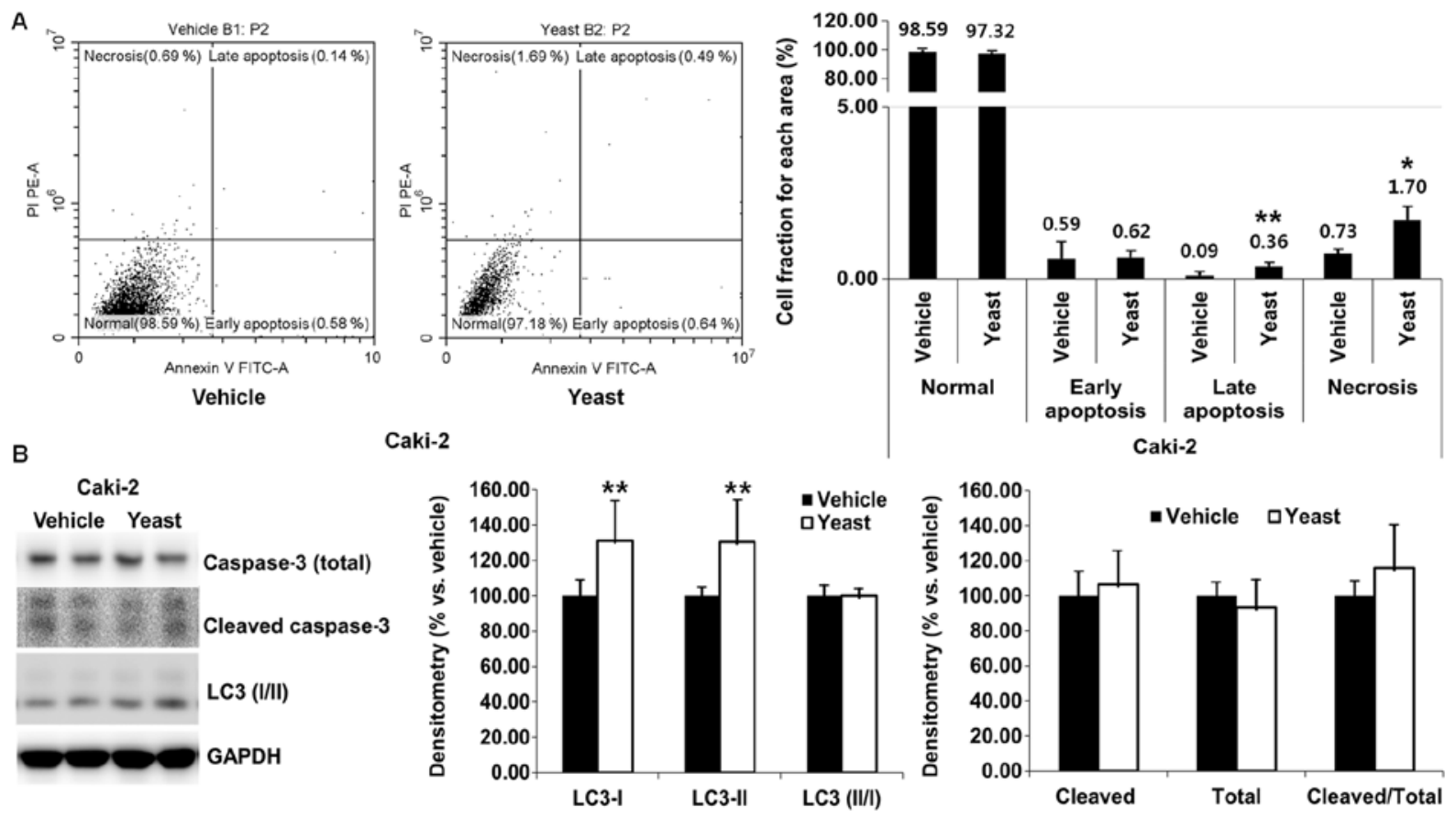

Figure 3. Yeast extract does not affect cell death signaling of primary RCC cells (Caki-2). (A) Annexin V/PI staining was used to assess the necrosis and apoptosis induced by $5.0 \%$ yeast extract in Caki-2 cells. Representative results of flow cytometric analysis of cell death distribution, in which the percentage of each quadrant is numerically indicated. Quantitative data are expressed as the mean \pm standard deviation. Late apoptosis and necrosis both significantly increased with relatively small percentages of the entire portions. (B) Western blot analysis revealed caspase-3 dependent apoptosis and LC3-dependent autophagy. There was no significant increase in cell death pathways, but there was a slight increase in cleaved caspase-3 vs. total caspase-3. Densitometry is presented vs. the vehicle-treated cells following treatment with yeast extract. Data are expressed as the mean \pm standard deviation. ${ }^{*} \mathrm{P}<0.05$ and ${ }^{* *} \mathrm{P}<0.01$ vs. vehicle. RCC, renal cell carcinoma.

A
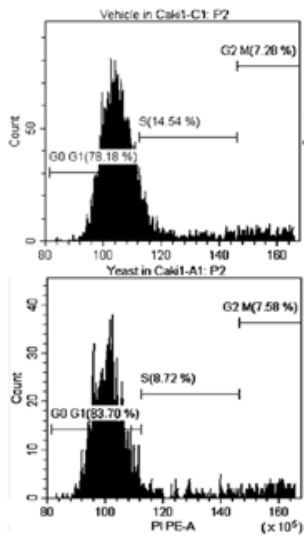

B

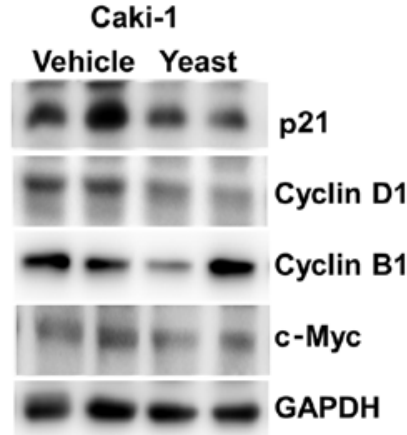

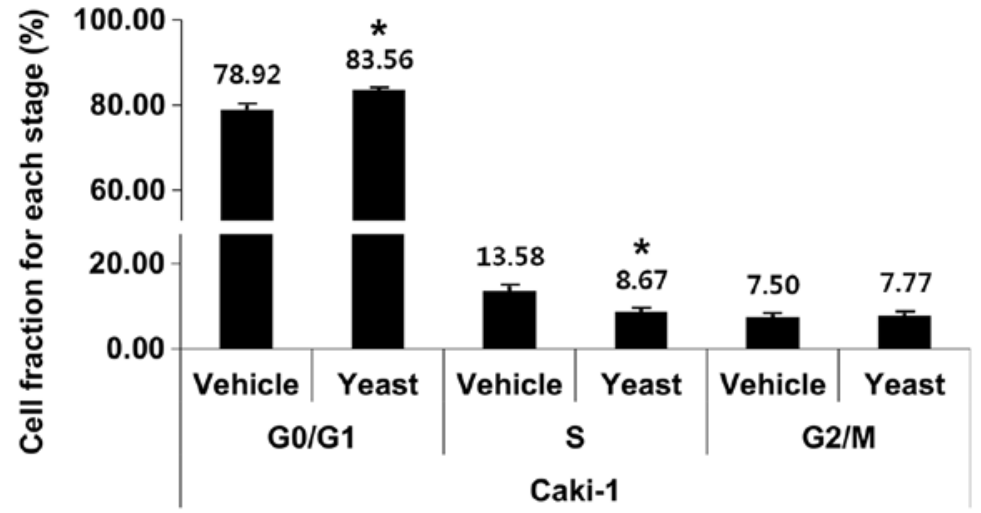

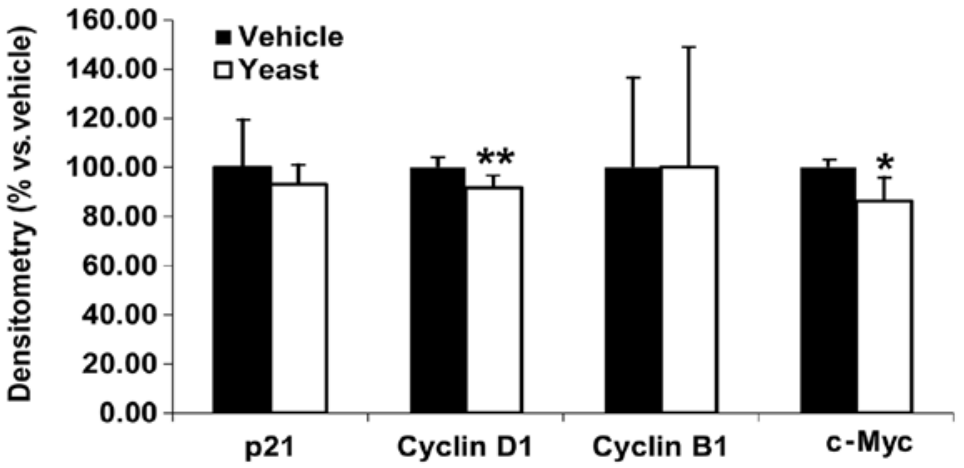

Figure 4. Yeast extract triggers cell cycle arrest at the $\mathrm{G}_{0} / \mathrm{G}_{1}$ phase in metastatic RCC cells (Caki-1). (A) Caki-1 cells were treated with yeast extract and cell cycle progression was monitored using flow cytometry. Representative results of flow cytometric analysis of cell cycle analysis, and quantitative data are expressed as the mean \pm standard deviation, revealing a significantly increased $\mathrm{G}_{0} / \mathrm{G}_{1}$ and decreased $\mathrm{S}$ fraction. (B) Western blot analysis revealed decreased p21, cyclin D1 and c-Myc following yeast extract treatment. Significance was observed in both c-Myc and cyclin D1, which may be a possible mechanism for $\mathrm{G}_{0} / \mathrm{G}_{1}$ arrest. Densitometry is presented vs. the vehicle-treated cells following treatment with yeast extract. Data are expressed as the mean \pm standard deviation. ${ }^{*} \mathrm{P}<0.05$ and ${ }^{* *} \mathrm{P}<0.01$ vs. vehicle. $\mathrm{RCC}$, renal cell carcinoma. 
A
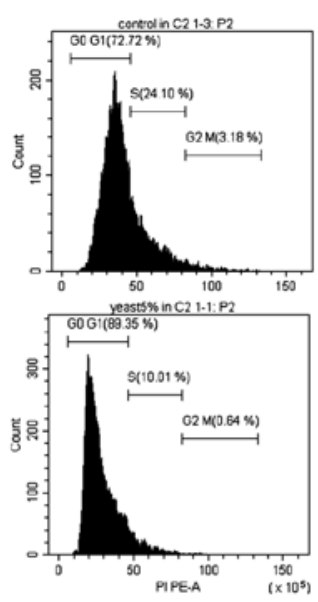

B

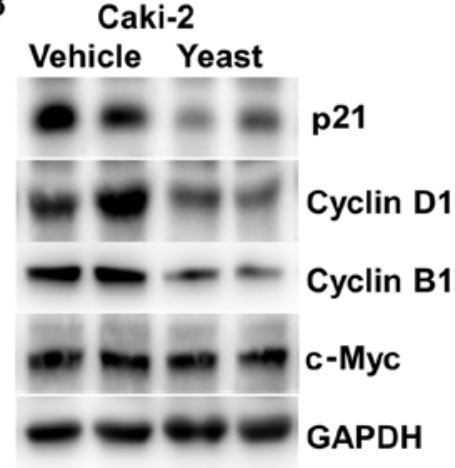

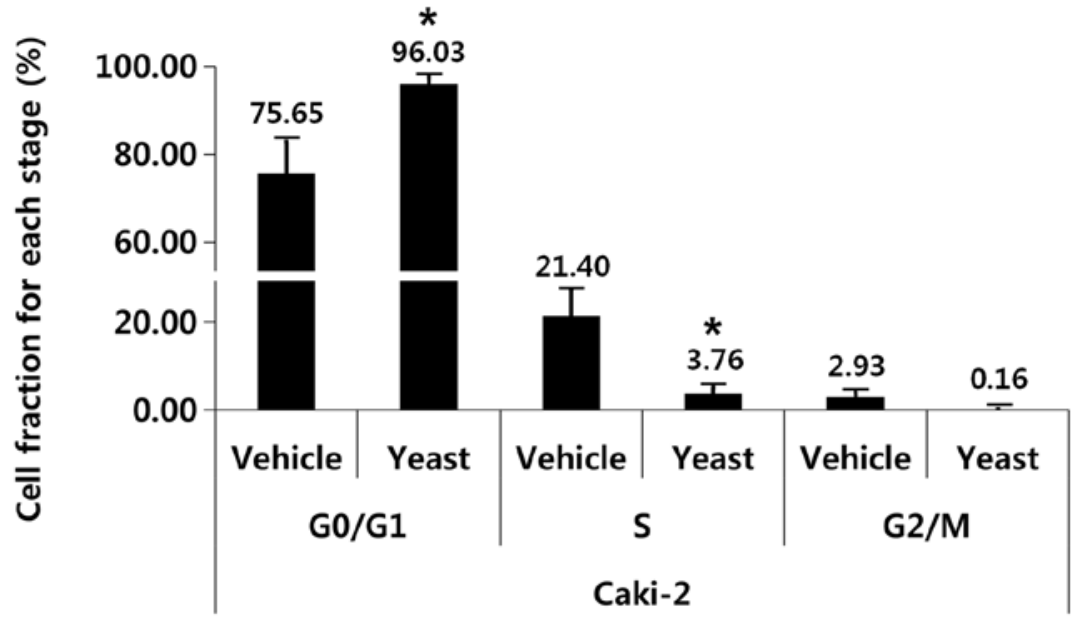

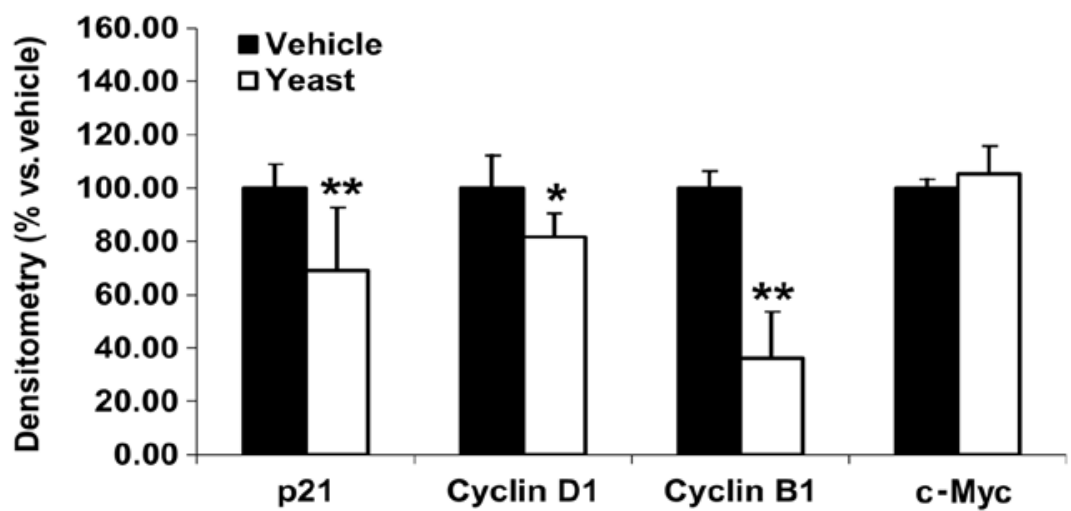

Figure 5. Yeast extract triggers cell cycle arrest at the $\mathrm{G}_{0} / \mathrm{G}_{1}$ phase in primary RCC cells (Caki-2). (A) Caki-2 cells were treated with yeast extract and cell cycle progression was monitored using flow cytometry. Representative results of flow cytometric analysis of cell cycle analysis, and quantitative data are expressed as the mean \pm standard deviation, revealing a significantly increased $\mathrm{G}_{0} / \mathrm{G}_{1}$ and decreased $\mathrm{S}$ fraction. (B) Western blot analysis revealed decreased p21, cyclin D1, and cyclin B1 following yeast extract treatment. Decreased cyclin D1 could be a possible mechanism for G0/G1 arrest, but decreased cyclin B1 may be involved in another mechanism for cell cycle arrest. Densitometry is presented vs. the vehicle-treated cells following treatment with yeast extract. Data are expressed as the mean \pm standard deviation. ${ }^{*} \mathrm{P}<0.05,{ }^{* *} \mathrm{P}<0.01$ vs. vehicle. $\mathrm{RCC}$, renal cell carcinoma.

CuZnSOD and MnSOD were revealed to be differentially altered. While CuZnSOD ( $\mathrm{P}=0.005$ in Caki-1, and $\mathrm{P}=0.010$ in Caki-2) and MnSOD ( $\mathrm{P}=0.048$ in Caki-1, and $\mathrm{P}=0.021$ in Caki-2) were significantly increased in both RCC cells, catalase was considerably decreased in Caki-1 $(\mathrm{P}<0.001$, Fig. 6B) but was not altered in Caki-2 (Fig. 6C) cells. Specifically, densitometry revealed $165.84 \pm 66.14$ and $153.31 \pm 55.81 \%$ of CuZnSOD, $110.95 \pm 8.40$ and $123.38 \pm 17.31 \%$ of MnSOD, and $60.12 \pm 12.61$ and $115.82 \pm 17.61 \%$ of catalase in Caki-1 and Caki-2 cells, respectively.

Yeast extract did affect cell proliferation via regulating the iron metabolism. Following yeast extract treatment, potential changes in antioxidant activity, GPX4, and related proteins which were related to iron metabolism were investigated in RCC cells.

The levels of GPX4, transferrin receptor (TfRC), ferritin heavy chain (FTH1), and cysteine/glutamate transporter (xCT) were revealed to be differentially altered (Fig. 7). While a significant increase of FTH1 $(\mathrm{P}<0.001$ in Caki-1, and $\mathrm{P}=0.005$ in Caki-2) was observed no change in $\mathrm{xCT}$ was observed in both RCC cell lines. In addition, a significant decrease of TfRC $(\mathrm{P}=0.001)$ was revealed in Caki-1 (Fig. 7B) and a significant decrease of GPX4 was revealed in Caki-2 cells $(\mathrm{P}=0.007$; Fig. 7C). Specifically, densitometry revealed $208.26 \pm 32.78$ and $179.43 \pm 55.78 \%$ of FTH1 in Caki-1 and Caki- 2 cells, respectively, along with $71.45 \pm 14.76 \%$ of TfRC in Caki-1 and $76.40 \pm 25.26 \%$ of GPX4 in Caki-2 cells.

\section{Discussion}

Based on the antitumor effects of RCM, six ingredients including yeast extract were assessed for their antitumor effects, and yeast extract was revealed to be the best candidate. According to the manufacturer (Sigma-Aldrich; Merck $\mathrm{KGaA}$ ), yeast extract (product no. Y1625) is a mixture of amino acids, peptides, water soluble vitamins (including B-complex vitamins), and carbohydrates, and it is suitable for use as a nutritional source in microbial culture media. Yeast extract has previously been revealed to inhibit mitosis of cancer cells while having no inhibitory effects on non-neoplastic cells (12-14). We first showed that yeast extract exhibited growth inhibition activity on RCC cells when compared with a vehicle (DW)-treated control, which had dose- and time-dependent anti-proliferative effects, and relatively slight growth inhibition activity in normal human proximal tubular cells. Since no characteristic features of necrosis, apoptosis, or autophagy were observed, the cancer cells may have been arrested at a certain phase of the cell 


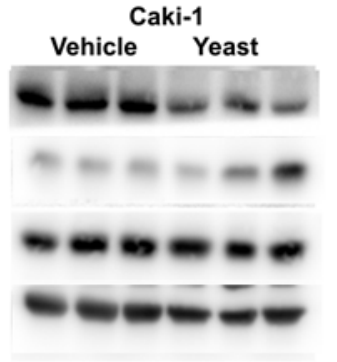

B

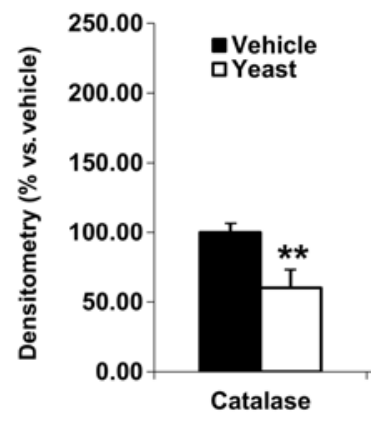

C

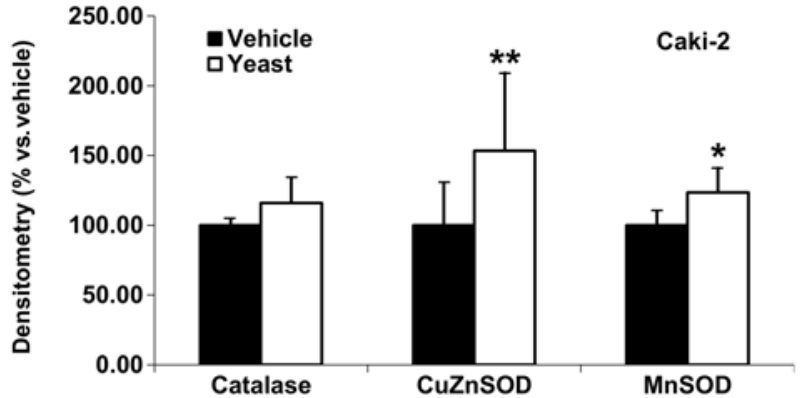

Figure 6. Yeast extract does affect intracellular antioxidant activities in RCC cells. (A) Western blot analysis revealed catalase, CuZnSOD and MnSOD activities after yeast extract treatment. The increased antioxidant activities may be involved in cell cycle arrest. (B) CuZnSOD and MnSOD were significantly increased, but catalase was significantly decreased in metastatic RCC cells (Caki-1). (C) All antioxidant enzymes assessed were increased in primary RCC cells (Caki-2). Densitometry is presented vs. the vehicle-treated cells following treatment with yeast extract. Data are expressed as the mean \pm standard deviation. ${ }^{*} \mathrm{P}<0.05,{ }^{* * *} \mathrm{P}<0.01$ vs. vehicle. $\mathrm{RCC}$, renal cell carcinoma.

cycle instead of moving to the sub- $G_{0} / G_{1}$ phase. Based on cell cycle analysis, $\mathrm{G}_{0} / \mathrm{G}_{1}$ arrest under yeast extract treatment was induced by the decreased cyclin D1 expression in RCC cells. Caki-2 exhibited a slower proliferation curve compared with Caki-1 cells (Fig. 1D), which would be related with the G1/S arrest. In addition, different cell cycle regulators may also be partially involved in the anti-proliferative effects of yeast extract including cyclin $\mathrm{B} 1$, the $\mathrm{G} 2 / \mathrm{M}$ phase regulator, particularly in Caki-2 cells. These results were not surprising due to the nature of the yeast extract of the mixture, but it was not considered to be a major mechanism for cell cycle arrest since anticancer drugs have exhibited cell cycle arrest with diminished cyclin D1 and/or cyclin B1 (15). The inhibition of tumor cell growth can also be attributed to the increase in the steady state levels of hydrogen peroxide caused by the increased activity of antioxidant enzymes (16). Yeast extract-treated cells exhibited higher levels of classic antioxidant activities with catalase, MnSOD and CuZnSOD, which may be responsible for the inhibition of cancer growth.

A
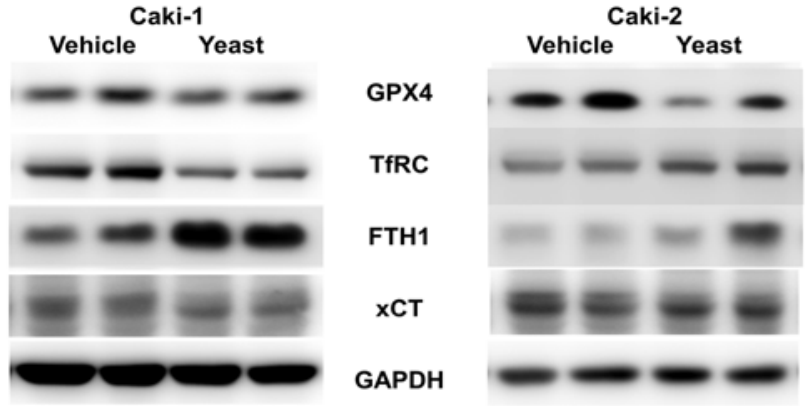

B

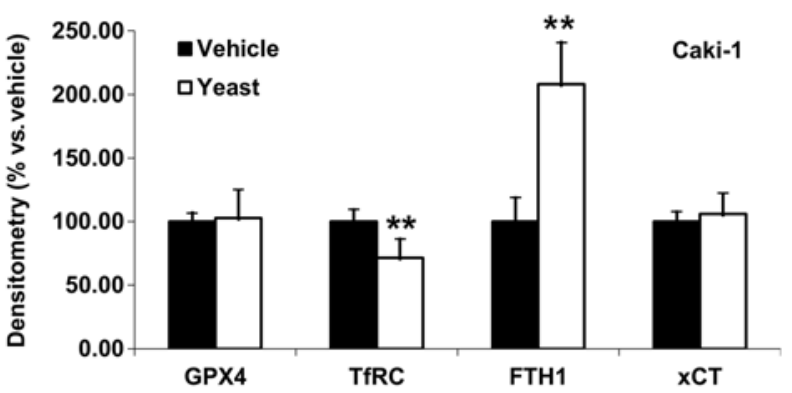

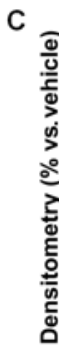

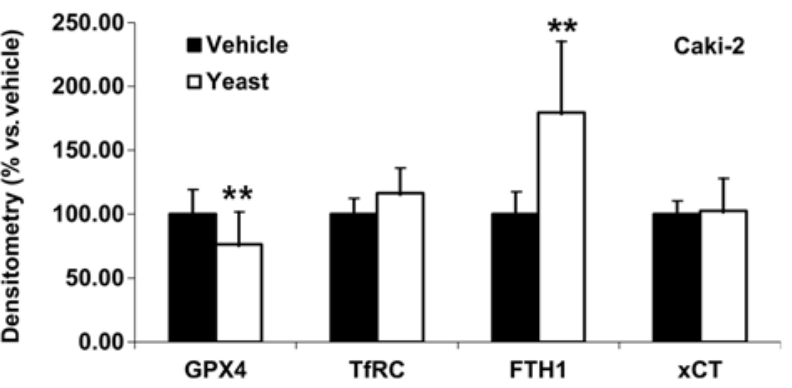

Figure 7. Yeast extract does affect iron metabolism and/or iron-dependent cell death. (A) Western blot analysis revealed GPX4, TfRC, FTH1 and $\mathrm{xCT}$ following yeast extract treatment. (B) Significance was observed with increased FTH1 and decreased TfRC, while GPX4 and $\mathrm{xCT}$ were not altered in metastatic RCC cells (Caki-1), which is responsible for the growth inhibition activity via low free iron in the cell. (C) Significance was observed with increased FTH1 and decreased GPX4, while the TfRC and $\mathrm{xCT}$ were not altered in primary RCC cells (Caki-2), which is responsible for the iron-dependent cell death, ferroptosis. Densitometry is presented vs. the vehicle-treated cells following treatment with yeast extract. Data are expressed as the mean \pm standard deviation. ${ }^{* *} \mathrm{P}<0.01$. TfRC, transferrin receptor; FTH1, ferritin heavy chain; $\mathrm{xCT}$, cysteine/glutamate transporter; RCC, renal cell carcinoma.

Although the active cell death was unchanged, LC3 was increased in Caki-2 and decreased in Caki-1 cells, indicating that different cell death pathways may exist in RCC cells, particularly in Caki-2 cells. Notably, GPX4, which belongs to the family of glutathione peroxidases, was significantly decreased in primary clear cell RCC cells (Caki-2). This is worth noting since the inactivation of GPX4 leads to an accumulation of lipid peroxides, resulting in ferroptosis, an iron-induced non-apoptotic and non-necrotic oxidative form of programmed cell death (17-21). Iron contributes to mutagenicity and malignant transformation, and then malignant cells require high amounts of iron for proliferation. For the high requirement of iron, TfRC and FTH1 were increased in transformed malignant cells $(22,23)$. Recently, changes in iron profile have been suggested as a successful marker for chemotherapy of metastatic renal cancer (24) based on previous 
accumulated studies involving serum iron (25), ferritin (26-28) or TfRC $(25,28)$. As ferroptosis is induced by the inhibition of cysteine uptake or the inactivation of the lipid repair enzyme GPX4 (29), xCT was further examined, but no significant changes were revealed in RCC cells. Yeast treatment led to decreased transferrin receptors and increased ferritin in metastatic RCC cells (Caki-1), which may partially contribute to the growth inhibition activity of yeast extract via the low free iron level in the cancer cells. However, a slight increase in transferrin receptors and a decreased GPX4 were observed in primary RCC cells (Caki-2), suggesting that ferroptosis may be involved in the antitumor effects of yeast extract in Caki-2 cells. This can be reinforced by the facts that targeted drugs on RCC, including tyrosine kinase (sorafenib and pazopanib) or mammalian target of rapamycin (mTOR) inhibitor (everolimus and temsirolimus), inhibit growth factors that have been revealed to promote the growth and spread of tumors (30). The tyrosine kinase, sorafenib, is also known as an inhibitor for cysteine transporter (20), and the mTOR pathway is one of the regulators of iron homeostasis via iron, ferritin, and TfRC $(24,28,31)$. However, recent molecular data (32) insists that Caki-2 is a type of papillary RCC rather than a clear cell RCC, which may account for the different responses in this experiment. More RCC cell lines, including clear cell or papillary cell types, should be included to determine the possible mechanism of antitumor effects of yeast extract in future experiments.

In conclusion, yeast extract, a mixture of compounds, may have a variety of effects on cancer cells requiring further investigation, however, herein its potential roles in the growth inhibition activities on RCC cells were clearly revealed. The anti-proliferative effects of yeast extract were iron-dependent and resulted in G0/G1 arrest through decreased cyclin D1 and increased cell death, possibly via ferroptosis in primary RCC cells. The iron-dependent cell death pathway may be another mechanism for the antitumor effects of yeast extract and should be further researched, particularly in primary RCC.

\section{Acknowledgements}

Parts of these data were presented at the 8th Asia Pacific International Congress of Anatomists, October 2018.

\section{Funding}

This study was supported by the Basic Science Research Program through the National Research Foundation of Korea (NRF) funded by the Ministry of Education (grant no. 2018R1D1A1A02050497).

\section{Availability of data and materials}

The datasets used during the present study are available from the corresponding author upon reasonable request.

\section{Authors' contributions}

SPY conceived and designed the present study, and wrote the manuscript. DM, JK and SPY performed the experiments for data acquisition and analysis. DM and SPY interpreted the experimental results. All authors read and approved the manuscript and agree to be accountable for all aspects of the research in ensuring that the accuracy or integrity of any part of the work are appropriately investigated and resolved.

\section{Ethics approval and consent to participate}

Not applicable.

\section{Patient consent for publication}

Not applicable.

\section{Competing interests}

The authors declare that they have no competing interests.

\section{References}

1. Inamura K: Renal cell tumors: Understanding their molecular pathological epidemiology and the 2016 WHO classification. Int J Mol Sci 18: pii: E2195, 2017.

2. Koo KC and Chung BH: Epidemiology and treatment patterns of urologic cancers in Korea. Korean J Urol Oncol 13: 51-57, 2015.

3. Rini BI, Rathmell WK and Godley P: Renal cell carcinoma. Curr Opin Oncol 20: 300-306, 2008.

4. Compare D and Nardone G: Contribution of gut microbiota to colonic and extracolonic cancer development. Dig Dis 29: 554-561, 2011.

5. Pope JL, Tomkovich S, Yang Y and Jobin C: Microbiota as a mediator of cancer progression and therapy. Transl Res 179: 139-154, 2017.

6. Ramezani A and Raj DS: The gut microbiome, kidney disease, and targeted interventions. J Am Soc Nephrol 25: 657-670, 2014.

7. Ramezani A, Massy ZA, Meijers B, Evenepoel P, Vanholder R and Raj DS: Role of the gut microbiome in uremia: A Potential therapeutic target. Am J Kidney Dis 67: 483-498, 2016.

8. Li L, Ma L and Fu P: Gut microbiota-derived short-chain fatty acids and kidney diseases. Drug Des Devel Ther 11: 3531-3542, 2017.

9. Chui CH, Cheng GY, Ke B, Lau FY, Wong RS, Kok SH, Fatima S, Cheung F, Cheng CH, Chan AS and Tang JC: Growth inhibitory potential of effective microorganism fermentation extract (EM-X) on cancer cells. Int J Mol Med 14: 925-929, 2004.

10. Yoon SP and Kim J: Exogenous CGRP upregulates profibrogenic growth factors through PKC/JNK signaling pathway in kidney proximal tubular cells. Cell Biol Toxicol 34: 251-262, 2018.

11. Kim JB, Hwang SE and Yoon SP: Dexamethasone reduces side population fraction through downregulation of ABCG2 transporter in MCF-7 breast cancer cells. Mol Med Rep 16: 453-458, 2017.

12. Okada $\mathrm{T}$ and Yoneyama M: The interaction of malignant cells with yeast. II. A new yeast extract inhibiting the growth of malignant cell. Hiroshima J Med Sci 19: 99-117, 1970.

13. Fardon JC, Poydock ME and Tsuchiya Y: Response of neoplastic and non-neoplastic cells to a yeast extract. J Surg Oncol 10: 10-21, 1978.

14. Poydock ME, Fardon JC, Tsuchiya Y and Cook ES: Selective effect of fractions of a yeast extract (PCO) on normal and malignant cells. Exp Cell Biol 46: 231-239, 1978.

15. Heffeter P, Jakupec MA, Körner W, Wild S, von Keyserlingk NG, Elbling L, Zorbas H, Korynevska A, Knasmüller S, Sutterlüty H, et al: Anticancer activity of the lanthanum compound [tris(1,10-phenanthroline)lanthanum(III)] trithiocyanate (KP772; FFC24). Biochem Pharmacol 71: 426-440, 2006.

16. Kim KH, Rodriguez AM, Carrico PM and Melendez JA: Potential mechanisms for the inhibition of tumor cell growth by manganese superoxide dismutase. Antioxid Redox Signal 3: 361-373, 2001.

17. Yang WS, SriRamaratnam R, Welsch ME, Shimada K, Skouta R, Viswanathan VS, Cheah JH, Clemons PA, Shamji AF, Clish CB, et al: Regulation of ferroptotic cancer cell death by GPX4. Cell 156: 317-331, 2014. 
18. Friedmann Angeli JP, Schneider M, Proneth B, Tyurina YY, Tyurin VA, Hammond VJ, Herbach N, Aichler M, Walch A, Eggenhofer E, et al: Inactivation of the ferroptosis regulator Gpx4 triggers acute renal failure in mice. Nat Cell Biol 16: 1180-1191, 2014.

19. Kazan HH, Urfali-Mamatoglu $\mathrm{C}$ and Gunduz U: Iron metabolism and drug resistance in cancer. Biometals 30: 629-641, 2017.

20. Yu H, Guo P, Xie X, Wang Y and Chen G: Ferroptosis, a new form of cell death, and its relationships with tumourous diseases. J Cell Mol Med 21: 648-657, 2017.

21. Martin-Sanchez D, Poveda J, Fontecha-Barriuso M, RuizAndres O, Sanchez-Niño MD, Ruiz-Ortega M, Ortiz A and Sanz AB: Targeting of regulated necrosis in kidney disease. Nefrologia 38: 125-135, 2018 (In English, Spanish).

22. Fanzani A and Poli M: Iron, oxidative damage and ferroptosis in rhabdomyosarcoma. Int J Mol Sci 18: pii: E1718, 2017.

23. Pfeifhofer-Obermair C, Tymoszuk P, Petzer V, Weiss G and Nairz M: Iron in the tumor microenvironment-connecting the dots. Front Oncol 8: 549, 2018.

24. Golčić $\mathrm{M}$ and Petković M: Changes in metabolic profile, iron and ferritin levels during the treatment of metastatic renal cancer-A new potential biomarker? Med Hypotheses 94: 148-150, 2016.

25. Yu CC, Chen KK, Chen MT, Huang JK, Lin AT, Lee YH and Chang LS: Serum iron as a tumor marker in renal cell carcinoma. Eur Urol 19: 54-58, 1991.
26. Partin AW, Criley SR, Steiner MS, Hsieh K, Simons JW, Lumadue J, Carter HB and Marshall FF: Serum ferritin as a clinical marker for renal cell carcinoma: Influence of tumor volume. Urology 45: 211-217, 1995.

27. Kirkali Z, Esen AA, Kirkali G and Güner G: Ferritin: A tumor marker expressed by renal cell carcinoma. Eur Urol 28: 131-134, 1995.

28. Torti SV and Torti FM: Ironing out cancer. Cancer Res 71: 1511-1514, 2011.

29. Latunde-Dada GO: Ferroptosis: Role of lipid peroxidation, iron and ferritinophagy. Biochim Biophys Acta Gen Subj 1861: 1893-1900, 2017.

30. Santoni M,De Tursi M,Felici A,Lo Re G, Ricotta R, Ruggeri EM, Sabbatini R, Santini D, Vaccaro V and Milella M: Management of metastatic renal cell carcinoma patients with poor-risk features: Current status and future perspectives. Expert Rev Anticancer Ther 13: 697-709, 2013.

31. Bayeva M, Khechaduri A, Puig S, Chang HC, Patial S, Blackshear PJ and Ardehali H: mTOR regulates cellular iron homeostasis through tristetraprolin. Cell Metab 16: 645-657, 2012.

32. Brodaczewska KK, Szczylik C, Fiedorowicz M, Porta C and Czarnecka AM: Choosing the right cell line for renal cell cancer research. Mol Cancer 15: 83, 2016. 